\title{
2019-nCoV Transmission in Hubei Province, China: Stochastic and Deterministic Analyses
}

\author{
Zhiming Li $\mathbb{D},{ }^{1}$ Zhidong Teng $\mathbb{D},{ }^{1}$ and Changxing $\mathrm{Ma}^{2}$ \\ ${ }^{1}$ College of Mathematics and System Sciences, Xinjiang University, Urumqi 834800, China \\ ${ }^{2}$ Department of Biostatistics, University at Buffalo, Buffalo 14214, USA \\ Correspondence should be addressed to Zhidong Teng; zhidong@xju.edu.cn
}

Received 12 February 2020; Revised 11 May 2020; Accepted 8 June 2020; Published 30 July 2020

Academic Editor: Chittaranjan Hens

Copyright (c) 2020 Zhiming Li et al. This is an open access article distributed under the Creative Commons Attribution License, which permits unrestricted use, distribution, and reproduction in any medium, provided the original work is properly cited.

Currently, a novel coronavirus (2019-nCoV) causes an outbreak of viral pneumonia in Hubei province, China. In this paper, stochastic and deterministic models are proposed to investigate the transmission mechanism of 2019-nCoV from 15 January to 5 February 2020 in Hubei province. For the deterministic model, basic reproduction number $R_{0}$ is defined and endemic equilibrium is given. Under $R_{0}>1$, quasi-stationary distribution of the stochastic process is approximated by Gaussian diffusion. Residual, sensitivity, dynamical, and diffusion analyses of the models are conducted. Further, control variables are introduced to the deterministic model and optimal strategies are provided. Based on empirical results, we suggest that the first and most important thing is to control input, screening, treatment, and isolation.

\section{Introduction}

On 11 January 2020, 41 cases of pneumonia with unknown causes were reported by Wuhan Municipal Health Commission [1]. The main clinical features initially include fever, cough, shortness of breath, or chest radiographs showing invasive pneumonic infiltrates in both lungs. Some patients subsequently developed pneumonia, acute respiratory distress syndrome, kidney failure, and even death [2, 3]. On 12 January 2020, the World Health Organization (WHO) termed it as 2019 novel coronavirus (2019-nCoV) [4]. The National Health Commission of China announced 2019$\mathrm{nCoV}$ infected pneumonia to be included in the management of statutory infectious diseases on 20 January 2020 [5]. On 11 February 2020, the WHO gave an official name to the novel coronavirus that has killed more than 1,000 people as coronavirus disease (COVID-19) [6].

The 2019-nCoV outbreak has received considerable attention from domestic and international scholars [7-13]. Clinical evidence of 2019-nCoV refers to [14-16]. Chan et al. [17] indicated 2019-nCoV disease can spread from person to person by a study of a family cluster. The authors of [18-20] analyzed transmission risk, the unreported number, and basic reproduction number estimations of 2019-nCoV cases in China, respectively. Mathematical modeling can be an important tool for gaining an understanding of the spread of 2019-nCoV such as stochastic and deterministic models. For stochastic versions of SI, SIS, SIR, and SIRS models, refer to $[21,22]$. Concerning the deterministic models, special attention has received those using ordinary differential equations and dynamical systems in their formulation [23, 24].

As of 5 February 2020, more than 28,000 cases with 2019$\mathrm{nCoV}$ and 600 deaths have been reported by the National Health Commission of China [5], and there were $70 \%$ of confirmed cases and $97 \%$ of deaths in Hebei province, China. In this paper, we propose a stochastic SEI process and a deterministic model to investigate 2019-nCoV transmission in Hubei province. We introduce an SEI Markovian epidemic process to analyze a density process in Section 2.1. A deterministic proportion model is proposed in Section 2.2, including an endemic equilibrium and local stability. A Gaussian diffusion approximation is considered in Section 2.3. If a scaled density process starts close to a deterministic equilibrium, this diffusion process is an Ornstein-Uhlenbeck $(\mathrm{O}-\mathrm{U})$ process. Main results are obtained in Section 3, and discussions are given in Section 4. 


\section{Methods}

Suppose that the host population $N$ is partitioned into three compartments: susceptible, exposed (infected but symptomfree), and infectious with symptoms. Let $S(t), E(t)$, and $I(t)$ be the numbers of susceptible, exposed, and infectious individuals in the population at time $t$, respectively. $E(t)+$ $I(t)$ is the total infected population. In the case of 2019$\mathrm{nCoV}$, there have been some clues suggesting that exposed individuals without symptom can cause many infections [25]. After one unit time, a susceptible individual can be infected through contacting with the exposed or infectious individuals and enter the $E$ class or is still in the $S$ class or dies. An exposed individual may have a symptom and enter the $I$ class or still stay in $E$ class or die. The dynamical transfer of the SEI process is demonstrated in Figure 1.

In Figure 1 , the parameter $\theta$ is the input rate in the susceptible group. Parameter $\mu$ denotes the natural death rate and $\mu_{1}$ is the rate of disease-caused death. The force of infection is $\beta_{1} E / N$ and $\beta_{2} I / N$, where $\beta_{1}$ and $\beta_{2}$ are defined as the effective contact per capita in the exposed and infectious periods. Thus, the incidence rate is $\left(\beta_{1} S E / N\right)+\left(\beta_{2} S I / N\right)$. Parameter $\sigma$ is a transfer rate from the exposed to infectious classes.

2.1. SEI Markov Process. For any $N>0$, we denote the process $\{(S(t), E(t), I(t)): t \geq 0\}$ with $N$ by a family of Markov chains $\left\{\left(S_{N}(t), E_{N}(t), I_{N}(t)\right): t \geq 0\right\}$ that take values in state space $\mathbb{E}_{N} \triangleq\{\mathbf{m}=(s, e, i), s, e, i=0,1,2, \ldots, N\}$ and have transition rate matrix $Q_{N} \triangleq\left(q_{N}(\mathbf{m}, \mathbf{n})\right)_{m, n \in \mathbb{E}_{N}}$ with $q_{N}(\mathbf{m}, \mathbf{n})$ representing the rate of transition from state $\mathbf{m}$ to $\mathbf{n}$ for $\mathbf{n} \neq \mathbf{m}$, and $q_{N}(\mathbf{m}, \mathbf{m}) \triangleq-q_{N}(\mathbf{m})$, where $q_{N}(\mathbf{m})=\sum_{n \neq m} q_{N}(\mathbf{m}, \mathbf{n})<\infty$. For convenience, denote $q_{N}(\mathbf{m}, \mathbf{n})=q_{N}(\mathbf{m}, \mathbf{m}+\mathbf{l})$ with $\mathbf{n}=$ $\mathbf{m}+\mathbf{l}$ and $\mathbf{l}=\left(l_{1}, l_{2}, l_{3}\right) \in L \triangleq\{(1,0,0),(-1,0,0),(-1,1,0)$, $(0,-1,0),(0,-1,1),(0,0,-1)\}$. From Figure 1, transition rates of the SEI process are defined by six events given in Table 1.

Consider all states of the SEI process conditioned on nonextinction state $I_{N}(t)>0$ at time $t$, denoted by $\left(S_{N}(t), E_{N}(t), I_{N}(t)\right) \mid I_{N}(t)>0$. Let $q_{s, e, i}(t)$ be condition probabilities and given by

$$
q_{s, e, i}(t)=P\left(\left(S_{N}(t), E_{N}(t), I_{N}(t)\right)=(s, e, i) \mid I_{N}(t)>0\right),
$$

where $s, e=0,1,2, \ldots, N, i=1,2, \ldots, N$. Such a distribution is called quasi-stationary distribution of the SEI process. For the SEI process, the set $\mathbb{E}_{N}$ of transient states is finite and irreducible. Thus, the quasi-stationary distribution exists and is unique [26].

Remark 1. According to the results of [21, 27], quasi-stationary distribution has different forms depending on the basic reproductive number $R_{0}$ and the expected population size $N$ in steady state. When $R_{0}$ is greater than 1 , the distribution can be approximated by Gaussian diffusion approximation. In this work, we mainly investigate that quasi-stationary distribution is approximately normal when $R_{0}>1$.
Denote

$$
\mathbf{X}_{N}(t)=\left(X_{1}(t), X_{2}(t), X_{3}(t)\right) \triangleq\left(\frac{S_{N}(t)}{N}, \frac{E_{N}(t)}{N}, \frac{I_{N}(t)}{N}\right) .
$$

It can be interpreted as the population densities of the susceptible, exposed, and infectious individuals at time $t$. Define a function $f: D\left(\subseteq \mathbb{R}_{+}^{3}\right) \times L \longrightarrow \mathbb{R} \triangleq(-\infty,+\infty)$ such that

$$
\begin{aligned}
f\left(\frac{\mathbf{m}}{N}, \mathbf{l}\right) & =\frac{1}{N} q_{N}(\mathbf{m}, \mathbf{m}+\mathbf{l}), \quad \frac{\mathbf{m}}{N} \in D, \mathbf{l} \in L, \\
F\left(\mathbf{X}_{N}\right) & =\sum_{l \neq 0} \mathbf{l} f\left(\mathbf{X}_{N}, \mathbf{l}\right),
\end{aligned}
$$

where $\mathbb{R}_{+}=(0, \infty)$. Therefore, $\left\{\mathbf{X}_{N}(t): t \geq 0\right\}$ is a density process with transition rate $f$ given in Table 1 . On the other hand, by the definition of $F$, we have

$$
\begin{aligned}
F\left(\mathbf{X}_{N}\right) \triangleq & \left(F_{1}\left(\mathbf{X}_{N}\right), F_{2}\left(\mathbf{X}_{N}\right), F_{3}\left(\mathbf{X}_{N}\right)\right) \\
= & \left(\theta-\beta_{1} X_{1} X_{2}-\beta_{2} X_{1} X_{3}-\mu X_{1}, \beta_{1} X_{1} X_{2}\right. \\
& \left.+\beta_{2} X_{1} X_{3}-(\mu+\sigma) X_{2}, \sigma X_{2}-\left(\mu+\mu_{1}\right) X_{3}\right) .
\end{aligned}
$$

For a density process, Ross et al. [28] can identify a deterministic analogue. Thus, the density process $\left\{\mathbf{X}_{N}(t): t \geq 0\right\}$ should behave more deterministically as $N$ becomes larger. The following theorem proves this point.

Theorem 1. Consider the density process $\left\{\mathbf{X}_{N}(t): t \geq 0\right\}$ defined in (2) and $\lim _{N \longrightarrow+\infty} \mathbf{X}_{N}(0)=\mathbf{x}(0) \triangleq \mathbf{X}_{0}$. Then, as $N \longrightarrow+\infty, \mathbf{X}_{N}(t)$ converges uniformly in probability over finite time intervals $[0, t]$ to a unique deterministic trajectory $\mathbf{x}(t)$ satisfying $\mathbf{x}(0)=\mathbf{x}_{0}, \mathbf{x}(s) \in D$ for $0 \leq s \leq t$ and

$$
\frac{\mathrm{d} \mathbf{x}(t)}{\mathrm{d} t}=F(\mathbf{x}(t)),
$$

where $\mathbf{x}(t)=\left(x_{1}(t), x_{2}(t), x_{3}(t)\right)$ and $F(\cdot)$ is defined in (4). That is to say, for fixed $t>0$ and for all $\epsilon>0$, $\lim _{N \rightarrow \infty} P\left(\sup _{s \leq t}\left|\mathbf{X}_{N}(s)-\mathbf{x}(s)\right|>\epsilon\right)=0$.

Proof. By definition of $f$ and Table 1 , it is clear that $f(\cdot, \cdot)$ is continuous for the first variable and therefore bounded over a compact set $K \subset \mathbb{R}_{+}^{3}$. Then, $\sup _{x \in D} \sum_{l}|\mathbf{l}| f(\mathbf{x}, \mathbf{l})<\infty$, $\mathbf{x} \in D, \mathbf{l} \in L$. On the other hand, every element of $F$ in (4) is continuous on any compact set $K$. Thus, $F$ is locally Lipschitz on $D$; that is, there exists a constant $C$ such that $|F(\mathbf{x})-F(\mathbf{y})|<C|\mathbf{x}-\mathbf{y}|, \mathbf{x}, \mathbf{y} \in D$. Based on Theorem 8.1 in the study of Krutz [29], the theorem follows.

Theorem 1 shows the relationship of density process $\left\{\mathbf{X}_{N}(t): t \geq 0\right\}$ and deterministic model (5). When $N$ is larger, we can investigate the properties of density process $\left\{\mathbf{X}_{N}(t): t \geq 0\right\}$ by those of $\mathbf{x}(t)$.

2.2. Deterministic Proportion Model. Denote $\mathbf{x}(t)=\left(x_{1}(t)\right.$, $\left.x_{2}(t), x_{3}(t)\right)$. For model (5), it is equal to the deterministic proportion model: 
$\left(\beta_{1} / N\right) S E+\left(\beta_{2} / N\right) S I$

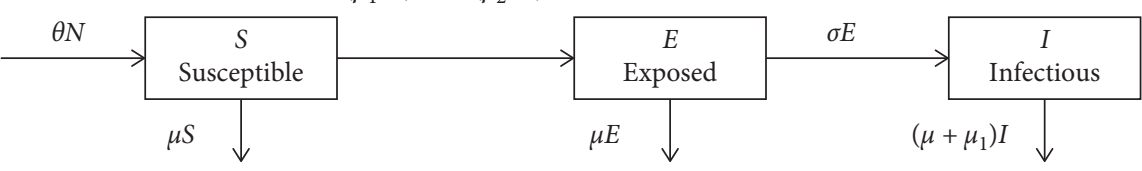

FIGURE 1: Transfer diagram of the SEI epidemic process.

TABLe 1: State transitions of $\left\{\left(S_{N}(t), E_{N}(t), I_{N}(t)\right): t \geq 0\right\}$ and $\left\{\mathbf{X}_{N}(t): t \geq 0\right\}$ at time $t$.

\begin{tabular}{lcccc}
\hline Event & State transition & $\mathbf{1}$ & $q_{N}(\mathbf{m}, \mathbf{m}+\mathbf{l})$ & $f\left(\left(X_{1}, X_{2}, X_{3}\right), \mathbf{l}\right)$ \\
\hline Input rate & $(s, e, i) \longrightarrow(s+1, e, i)$ & $(1,0,0)$ & $\theta N$ & $\theta$ \\
Death of susceptible & $(s, e, i) \longrightarrow(s-1, e, i)$ & $(-1,0,0)$ & $\mu s$ & $\mu X_{1}$ \\
Infection of susceptible & $(s, e, i) \longrightarrow(s-1, e+1, i)$ & $(-1,1,0)$ & $\left(\beta_{1} / N\right) s e+\left(\beta_{2} / N\right) s i$ & $\beta_{1} X_{1} X_{2}+\beta_{2} X_{1} X_{3}$ \\
Death of exposed & $(s, e, i) \longrightarrow(s, e-1, i)$ & $(0,-1,0)$ & $\mu e$ & $\mu X_{2}$ \\
Transfer rate & $(s, e, i) \longrightarrow(s, e-1, i+1)$ & $(0,-1,1)$ & $\sigma e$ & $\sigma X_{2}$ \\
Death of infected & $(s, e, i) \longrightarrow(s, e, i-1)$ & $(0,0,-1)$ & $\left(\mu+\mu_{1}\right) i$ & $\left(\mu+\mu_{1}\right) X_{3}$ \\
\hline
\end{tabular}

Note: $X_{1}=(s / N), X_{2}=(e / N)$, and $X_{3}=(i / N)$.

$$
\left\{\begin{array}{l}
\frac{\mathrm{d} x_{1}(t)}{\mathrm{d} t}=\theta-\beta_{1} x_{1}(t) x_{2}(t)-\beta_{2} x_{1}(t) x_{3}(t)-\mu x_{1}(t), \\
\frac{\mathrm{d} x_{2}(t)}{\mathrm{d} t}=\beta_{1} x_{1}(t) x_{2}(t)+\beta_{2} x_{1}(t) x_{3}(t)-(\mu+\sigma) x_{2}(t), \\
\frac{\mathrm{d} x_{3}(t)}{\mathrm{d} t}=\sigma x_{2}(t)-\left(\mu+\mu_{1}\right) x_{3}(t) .
\end{array}\right.
$$

However, model (6) has no explicit solution. Thus, we discuss the local stability of equilibrium by analyzing its characteristic equations. Model (6) has an endemic equilibrium denoted by $\mathbf{x}^{*}=\left(x_{1}^{*}, x_{2}^{*}, x_{3}^{*}\right)$, satisfying

$$
\left\{\begin{array}{l}
\theta-\beta_{1} x_{1}^{*} x_{2}^{*}-\beta_{2} x_{1}^{*} x_{3}^{*}-\mu x_{1}^{*}=0, \\
\beta_{1} x_{1}^{*} x_{2}^{*}+\beta_{2} x_{1}^{*} x_{3}^{*}-(\mu+\sigma) x_{2}^{*}=0, \\
\sigma x_{2}^{*}-\left(\mu+\mu_{1}\right) x_{3}^{*}=0
\end{array}\right.
$$

And by calculation, the endemic equilibrium $\mathbf{x}^{*}=\left(x_{1}^{*}, x_{2}^{*}, x_{3}^{*}\right)$ is obtained as follows:

$$
\left\{\begin{array}{l}
x_{1}^{*}=\frac{(\mu+\sigma)\left(\mu+\mu_{1}\right)}{\beta_{1}\left(\mu+\mu_{1}\right)+\beta_{2} \sigma}, \\
x_{2}^{*}=\frac{\theta\left(\beta_{1}\left(\mu+\mu_{1}\right)+\beta_{2} \sigma\right)-\mu(\mu+\sigma)\left(\mu+\mu_{1}\right)}{(\mu+\sigma)\left(\beta_{1}\left(\mu+\mu_{1}\right)+\beta_{2} \sigma\right)}, \\
x_{3}^{*}=\frac{\sigma\left(\theta\left(\beta_{1}\left(\mu+\mu_{1}\right)+\beta_{2} \sigma\right)-\mu(\mu+\sigma)\left(\mu+\mu_{1}\right)\right)}{(\mu+\sigma)\left(\mu+\mu_{1}\right)\left(\beta_{1}\left(\mu+\mu_{1}\right)+\beta_{2} \sigma\right)} .
\end{array}\right.
$$

Define the basic reproduction number as follows:

$$
R_{0}=\frac{\theta\left(\beta_{1}\left(\mu+\mu_{1}\right)+\beta_{2} \sigma\right)}{\mu(\mu+\sigma)\left(\mu+\mu_{1}\right)} \text {. }
$$

Theorem 2. If $R_{0}>1$, then the endemic equilibrium $\mathbf{x}^{*}=$ $\left(x_{1}^{*}, x_{2}^{*}, x_{3}^{*}\right)$ is locally asymptotically stable.

Proof. The Jacobian of model (6) at point $\mathbf{x}^{*}$ is given by

$$
J\left(\mathbf{x}^{*}\right)=\partial F\left(\mathbf{x}^{*}\right)=\left(\begin{array}{ccc}
-\beta_{1} x_{2}^{*}-\beta_{2} x_{3}^{*}-\mu & -\beta_{1} x_{1}^{*} & -\beta_{2} x_{1}^{*} \\
\beta_{1} x_{2}^{*}+\beta_{2} x_{3}^{*} & \beta_{1} x_{1}-(\mu+\sigma) & \beta_{2} x_{1}^{*} \\
0 & \sigma & -\left(\mu+\mu_{1}\right)
\end{array}\right) .
$$

Its characteristic equation is given as follows:

$$
\begin{aligned}
f(\lambda) & \triangleq \operatorname{det}\left(\lambda I-J\left(\mathbf{x}^{*}\right)\right) \\
& =\left|\begin{array}{ccc}
\lambda+\beta_{1} x_{2}^{*}+\beta_{2} x_{3}^{*}+\mu & \beta_{1} x_{1}^{*} & \beta_{2} x_{1}^{*} \\
-\beta_{1} x_{2}^{*}-\beta_{2} x_{3}^{*} & \lambda-\beta_{1} x_{1}^{*}+\mu+\sigma & -\beta_{2} x_{1}^{*} \\
0 & -\sigma & \lambda+\mu+\mu_{1}
\end{array}\right| \\
& =\lambda^{3}+a_{1} \lambda^{2}+a_{2} \lambda+a_{3},
\end{aligned}
$$

where

$$
\begin{aligned}
a_{1}= & \beta_{1} x_{2}^{*}+\beta_{2} x_{3}^{*}-\beta_{1} x_{1}^{*}+3 \mu+\mu_{1}+\sigma, \\
a_{2}= & \left(2 \mu+\mu_{1}+\sigma\right)\left(\beta_{1} x_{2}^{*}+\beta_{2} x_{3}^{*}\right)-\left(\beta_{1}\left(2 \mu+\mu_{1}\right)+\beta_{2} \sigma\right) x_{1}^{*} \\
& +2 \mu\left(\mu_{1}+\sigma\right)+\mu_{1} \sigma+3 \mu^{2}, \\
a_{3}= & \left(\mu\left(\mu+\mu_{1}+\sigma\right)+\mu_{1} \sigma\right)\left(\beta_{1} x_{2}^{*}+\beta_{2} x_{3}^{*}+\mu\right) \\
& -\mu\left(\beta_{1}\left(\mu+\mu_{1}\right)+\beta_{2} \sigma\right) x_{1}^{*} .
\end{aligned}
$$

Since $R_{0}>1$ and $x_{i}^{*}>0(i=1,2,3)$, by (8), we have

$$
\begin{aligned}
a_{1}= & \beta_{1} x_{2}^{*}+\beta_{2} x_{3}^{*}+\frac{\beta_{2} \sigma(\mu+\sigma)}{\beta_{1}\left(\mu+\mu_{1}\right)+\beta_{2} \sigma}+2 \mu+\mu_{1}>0, \\
a_{2}= & \left(2 \mu+\mu_{1}+\sigma\right)\left(\beta_{1} x_{2}^{*}+\beta_{2} x_{3}^{*}\right) \\
& +\frac{\beta_{1} \mu\left(\mu+\mu_{1}\right)^{2}+\beta_{2} \sigma \mu\left(\mu_{1}+2 \mu\right)}{\beta_{1}\left(\mu+\mu_{1}\right)+\beta_{2} \sigma}>0, \\
a_{3}= & \left(\mu\left(\mu+\mu_{1}+\sigma\right)+\mu_{1} \sigma\right)\left(\beta_{1} x_{2}^{*}+\beta_{2} x_{3}^{*}\right)>0 .
\end{aligned}
$$

Denote $A=\beta_{1} x_{2}^{*}+\beta_{2} x_{3}^{*}$. Then, 


$$
\begin{aligned}
a_{1} a_{2}-a_{3} & >\left(2 \mu+\mu_{1}\right)\left(2 \mu+\mu_{1}+\sigma\right) A-\left(\mu\left(\mu+\mu_{1}+\sigma\right)+\mu_{1} \sigma\right) A \\
= & \left(3 \mu\left(\mu+\mu_{1}\right)+\mu \sigma+\mu_{1}^{2}\right)>0 .
\end{aligned}
$$

According to Hurwitz criterion, all roots of $f(\lambda)$ have negative real parts. Then, the endemic equilibrium $\mathbf{x}^{*}$ is local asymptotic stability.

Remark 2. For $t \longrightarrow+\infty$ and larger number $N, \mathbf{X}_{N}(t)$ converges uniformly in probability to the endemic equilibrium $\mathbf{x}^{*}$; that is, $\left(S_{N}(t), E_{N}(t), I_{N}(t)\right)$ converges uniformly in probability to $\left(N x_{1}^{*}, N x_{2}^{*}, N x_{3}^{*}\right)$ in the endemic phase.

2.3. Diffusion Approximation. Deterministic model (6) is clearly an approximation of the density process $\left\{\mathbf{X}_{N}(t): t \geq 0\right\}$ in the endemic phase for $R_{0}>1$ (see Remark 2 ). However, it cannot reflect the fluctuations of the process around the endemic equilibrium $\mathbf{x}^{*}$. Gaussian diffusion approximation is used to analyze quasi-stationary distribution based on the work described in $[29,30]$. For convenience, define

$$
z_{N}(t)=\sqrt{N}\left(\mathbf{X}_{N}(t)-\mathbf{x}(t)\right) .
$$

The process $\left\{z_{N}(t): t \geq 0\right\}$ is called a scaled density process. Under certain conditions, $z_{N}(t)$ converges weakly in the space of all sample paths to a Gaussian diffusion with the initial value.

Theorem 3. Consider the process $\left\{z_{N}(t): t \geq 0\right\}$ and $\lim _{N \longrightarrow+\infty} \sqrt{N}\left(\mathbf{X}_{N}(0)-\mathbf{X}_{0}\right)=z$ (exist $)$. Then, $z_{N}(t)$ converges weakly to Gaussian diffusion $z(t)$ for $N \longrightarrow+\infty$, satisfying

$$
\mathrm{d} z(t)=\partial F\left(\mathbf{X}_{N}(t)\right) z(t) \mathrm{d} t+\sqrt{G\left(\mathbf{X}_{N}(t)\right)} \mathrm{d} w(t),
$$

with initial value $z$, where $\{w(t): t \geq 0\}$ is three-dimensional Wiener process and

$$
\begin{aligned}
G\left(\mathbf{X}_{N}\right) & \triangleq\left(\sum_{l} l_{i} l_{j} f\left(\mathbf{X}_{N}, \mathbf{l}\right)\right) \\
& =\left(\begin{array}{ccc}
\theta+\beta_{1} X_{1} X_{2}+\beta_{2} X_{1} X_{3}+\mu X_{1} & -\beta_{1} X_{1} X_{2}-\beta_{2} X_{1} X_{3} & 0 \\
-\beta_{1} X_{1} X_{2}-\beta_{2} X_{1} X_{3} & \beta_{1} X_{1} X_{2}+\beta_{2} X_{1} X_{3}+(\mu+\sigma) X_{2} & -\sigma X_{2} \\
0 & -\sigma X_{2} & \sigma X_{2}+\left(\mu+\mu_{1}\right) X_{3}
\end{array}\right),
\end{aligned}
$$

for $\mathbf{X}_{N}(t)=\left(X_{1}(t), X_{2}(t), X_{3}(t)\right) \in D$ and $\mathbf{1} \in L$.

Proof. From Theorem 1, trajectory of $\mathbf{x}(t)$ is contained in some compact set for $N \longrightarrow+\infty$ because of the local asymptotic stability of the endemic equilibrium $\mathbf{x}^{*}$. Therefore, $\partial F(\mathbf{x})$ is continuous and bounded to a compact set. On the other hand, by Table 1 and definition of $G(\mathbf{x}(t))$, equation (17) yields. Further, we know that $f(\mathbf{x}, \mathbf{l})$ is continuous in the first variable and bounded to a compact set. Then, for $\mathbf{l} \in L$, we have $\sup _{x \in D} \sum_{l}|\mathbf{1}|^{2} f(\mathbf{x}, \mathbf{l})<\infty$. By Theorem 8.2 given by Kurtz in [29], the result follows.

Theorem 3 reveals that the scaled process $\left\{z_{N}(t): t \geq 0\right\}$ can be approximated by Gaussian diffusion $z(t)$ for larger $N$. From Theorem 2, we get $\partial F\left(\mathbf{X}_{N}(t)\right) \longrightarrow \partial F\left(\mathbf{x}^{*}\right)$ and $G\left(\mathbf{X}_{N}(t)\right) \longrightarrow G\left(\mathbf{x}^{*}\right)$ as $t \longrightarrow+\infty$ so that $z(t)$ follows a stationary $\mathrm{O}-\mathrm{U}$ process given as a solution to

$$
\mathrm{d} z(t)=\partial F\left(\mathbf{x}^{*}\right) z(t) \mathrm{d} t+\sqrt{G\left(\mathbf{x}^{*}\right)} \mathrm{d} w(t),
$$

with the initial value $z$. The stationary distribution of $z(t)$ is multivariate normal with mean $\mathbf{0}$ and covariance matrix $\Sigma=$ $\left(\sigma_{i j}\right)_{3 \times 3}$ satisfying

$$
\partial F\left(\mathbf{x}^{*}\right) \Sigma+\Sigma \partial F\left(\mathbf{x}^{*}\right)^{T}=-G\left(\mathbf{x}^{*}\right) .
$$

From the above results, we know that the $\mathrm{O}-\mathrm{U}$ process $\{z(t): t \geq 0\}$ has stationary mean $\mathbf{0}$ and covariance matrix $\Sigma$ in the asymptotically stable case; that is, $z(t)=\sqrt{N}$
$\left(\mathbf{X}_{N}(t)-\mathbf{x}^{*}\right) \sim N(0, \Sigma)$. Then, the density process $\left\{\mathbf{X}_{N}(t): t \geq 0\right\}$ can be approximated by multivariate normal distribution $N\left(\mathbf{x}^{*},(1 / N) \Sigma\right)$ for larger number $N$ and $t \longrightarrow+\infty$. That is to say,

$$
\mathbf{X}_{N}(t)=\left(\frac{S_{N}(t)}{N}, \frac{E_{N}(t)}{N}, \frac{I_{N}(t)}{N}\right) \mid I_{N}(t)>0 \sim N\left(\mathbf{x}^{*}, \frac{1}{N} \Sigma\right),
$$

for $R_{0}>1$, which is diffusion approximation of the quasistationarity distribution $q_{s, e, i}$. Further, we have

$$
\left(S_{N}(t), E_{N}(t), I_{N}(t)\right) \mid I_{N}(t)>0 \sim N\left(N \mathbf{x}^{*}, N \Sigma\right) .
$$

\section{Main Results}

In this section, deterministic model (6) and stochastic model (21) are applied to analyze the characteristics of 2019-nCoV epidemic in Hubei province, China. The onset and death data were collected from Hubei from 15 January to 5 February $2020[31,32]$ (see Figure 2). The unknown parameters of models are estimated by least-squares method.

The spread of 2019-nCoV started in December 2019, in which the whole population of Hubei was 59,170,000; that is, $N=59,170,000$. For model $(6), x_{i}(t)(i=1,2,3)$ are the 


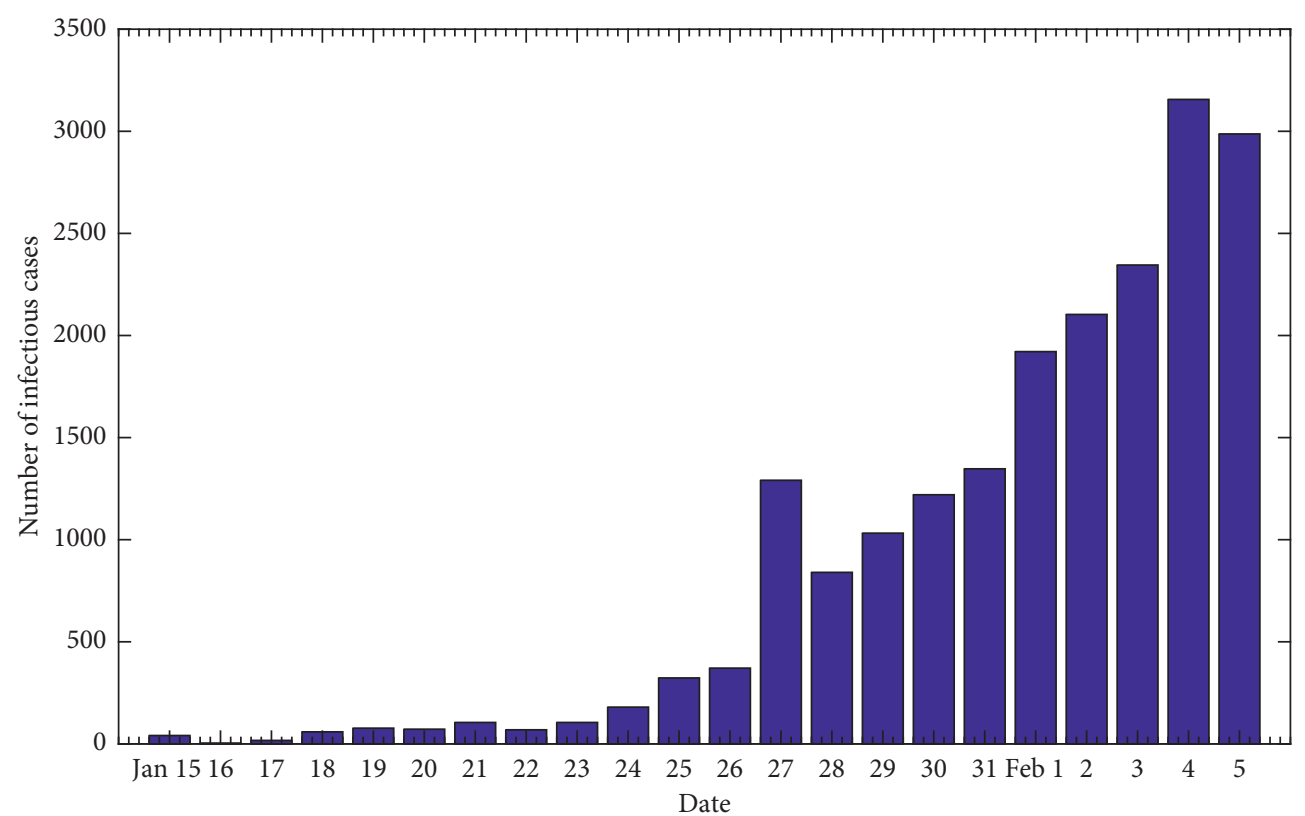

(a)

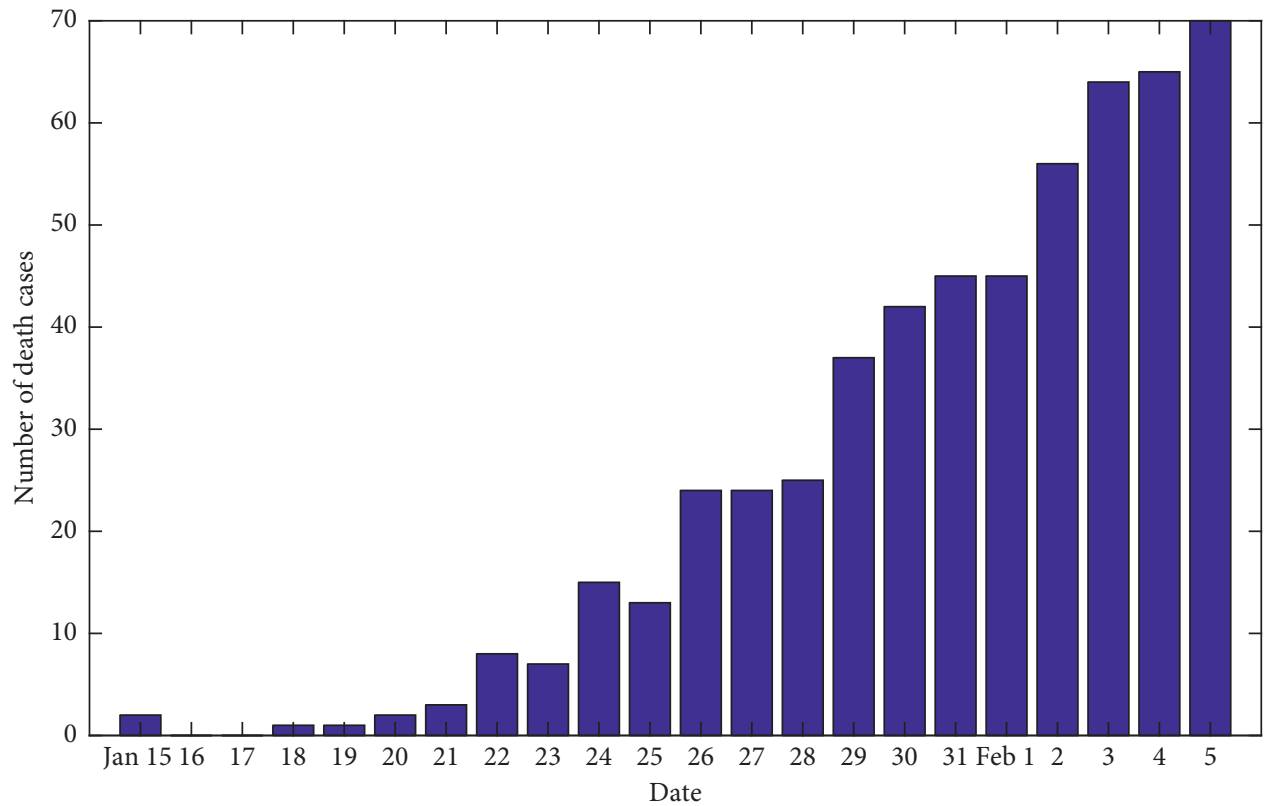

(b)

Figure 2: Numbers of confirmed and death cases in Hubei province, from 15 January 2020 to 5 February 2020.

population proportions of susceptible, exposed, and infectious individuals of Hubei province. From Figure 2 and [32], we have $I(0)=41$ and $E(0)=119$. Obviously, $S(0)=$ $N-I(0)-E(0)=59,169,840$. Thus,

$$
\begin{aligned}
& x_{1}(0)=\frac{S(0)}{N}=0.99999, \\
& x_{2}(0)=\frac{E(0)}{N}=2.011 \times 10^{-6}, \\
& x_{3}(0)=\frac{I(0)}{N}=6.929 \times 10^{-7} .
\end{aligned}
$$

Let $\widehat{\theta}, \widehat{\beta}_{i}(i=1,2), \widehat{\sigma}, \widehat{\mu}$, and $\widehat{\mu}_{1}$ be the estimated values of unknown parameters $\theta, \beta_{i}(i=1,2), \sigma, \mu$, and $\mu_{1}$, respectively. The estimated values are obtained by least-squares method (Table 2) with the initial values $x_{i}(0)(i=1,2,3)$. From Table 2, we have $R_{0}=\left(\left(\hat{\theta}\left(\widehat{\beta}_{1}\left(\widehat{\mu}+\widehat{\mu}_{1}\right)+\widehat{\beta}_{2} \widehat{\sigma}\right)\right) /(\widehat{\mu}(\widehat{\mu}+\right.$ $\left.\left.\widehat{\sigma})\left(\widehat{\mu}+\widehat{\mu}_{1}\right)\right)\right)=4.2656 \quad$ and $\quad x_{1}^{*}=0.1139$, $x_{2}^{*}=0.0046$, and $x_{3}^{*}=0.0734$. Since $R_{0}>1$, by Theorem 2 , the endemic equilibrium $\mathbf{x}^{*}=\left(x_{1}^{*}, x_{2}^{*}, x_{3}^{*}\right)$ is locally asymptotically stable. Further, it reveals that 2019-nCoV infection is still spreading and will be endemic in Hubei province without effective controls. Under model (6), Figure 3 shows the fitted values of susceptible, exposed, and 
TABLE 2: The estimated values of model parameters by least-squares method.

\begin{tabular}{lccc}
\hline Parameters & Definition & Initial values & Estimated values \\
\hline$\theta$ & Input rate into the susceptible group & 0.003 & 0.0034 \\
$\beta_{1}$ & Contact rate in the exposed period & 0.478 & 0.2800 \\
$\beta_{2}$ & Contact rate in the infectious period & 0.368 & 0.2936 \\
$\sigma$ & Transfer rate from exposed to the infectious period & 0.500 & 0.5554 \\
$\mu_{1}$ & Disease-caused death rate in the infectious period & 0.023 & 0.0280 \\
$\mu$ & Natural death rate & - & $0.007^{*}$ \\
\hline
\end{tabular}

${ }^{*}$ The estimation of $\mu$ is from [33].

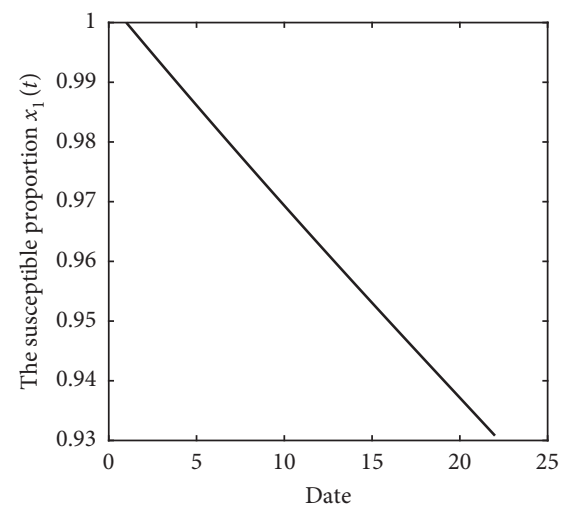

(a)

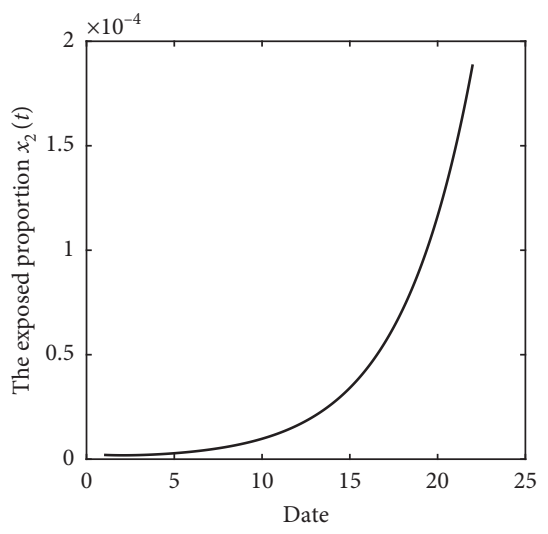

(b)

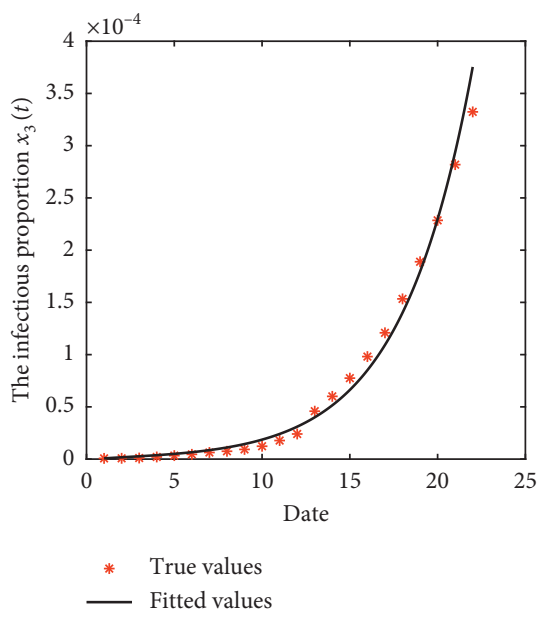

(c)

Figure 3: (a) Fitted curves of $x_{1}(t)$, (b) fitted curves of $x_{2}(t)$, and (c) true and fitted curves of $x_{3}(t)$ in Hubei province, from 15 January 2020 to 5 February 2020.

infectious proportions, comparing the actual values of infectious proportions.

3.1. Residual Analysis. Model validation is the most important step in the model building process. Residual analysis such as the coefficient of determination $R^{2}$ and mean squared error (MSE) provides measures of model quality. Figure 4 displays an error bar plot of the confidence intervals on the residuals of true and fitted values. As shown in Figure 4, residuals appear to behave randomly and the errors are relatively small for model (6). Therefore, it is reasonable to suggest that the estimators obtained here are reliable.

Let $\hat{x}_{3}(t)$ be the fitted value of $x_{3}(t)$ and $M$ be the number of observational data. Define

$$
\begin{aligned}
R^{2} & =1-\frac{\sum_{i=1}^{M}\left(x_{3}\left(t_{i}\right)-\widehat{x}_{3}\left(t_{i}\right)\right)^{2}}{\sum_{i=1}^{M}\left(x_{3}\left(t_{i}\right)-\bar{x}_{3}\left(t_{i}\right)\right)^{2}}, \\
\mathrm{MSE} & =\frac{1}{M} \sum_{i=1}^{M}\left(x_{3}\left(t_{i}\right)-\widehat{x}_{3}\left(t_{i}\right)\right)^{2},
\end{aligned}
$$

where $\hat{x}_{3}\left(t_{i}\right)=(1 / M) \sum_{i=1}^{M} x_{3}\left(t_{i}\right)$. From Figure 4 and the definitions of $R^{2}$ and MSE, we have $R^{2}=0.988$ and
MSE $=1.358 \times 10^{-10}$. Thus, model (6) can reflect the dynamics behavior of field data used in our study.

3.2. Sensitivity Analysis. Basic reproduction number $R_{0}$ is an important threshold value for the spread of 2019-nCoV and closely related to parameters $\theta, \beta_{i}(i=1,2), \sigma, \mu_{1}$, and $\mu$. Given all other parameters, Figure 5 shows that $R_{0}$ increases if $\theta, \beta_{1}$, or $\beta_{2}$ is larger, but it will decrease if $\sigma, \mu_{1}$, or $\mu$ increases.

Due to uncertainty associated with the estimation of certain parameter values, it is useful to carry out sensitivity analysis to investigate how sensitive $R_{0}$ is with respect to these parameters. The sensitivity index of $R_{0}$ that depends differentially on parameter $\tau$ is defined as follows:

$$
\zeta_{\tau}=\frac{\tau}{R_{0}} \frac{\mathrm{d} R_{0}}{\mathrm{~d} \tau}
$$

where $\tau=\theta, \beta_{i}(i=1,2), \sigma, \mu_{1}$, and $\mu$, and the quotient $\tau / R_{0}$ is introduced to normalize the index by removing the effects of units. The sensitivity index is basically the ratio of the change in output to the change in input while all other parameters remain constant. By calculation, we get the sensitivity indexes as follows: 


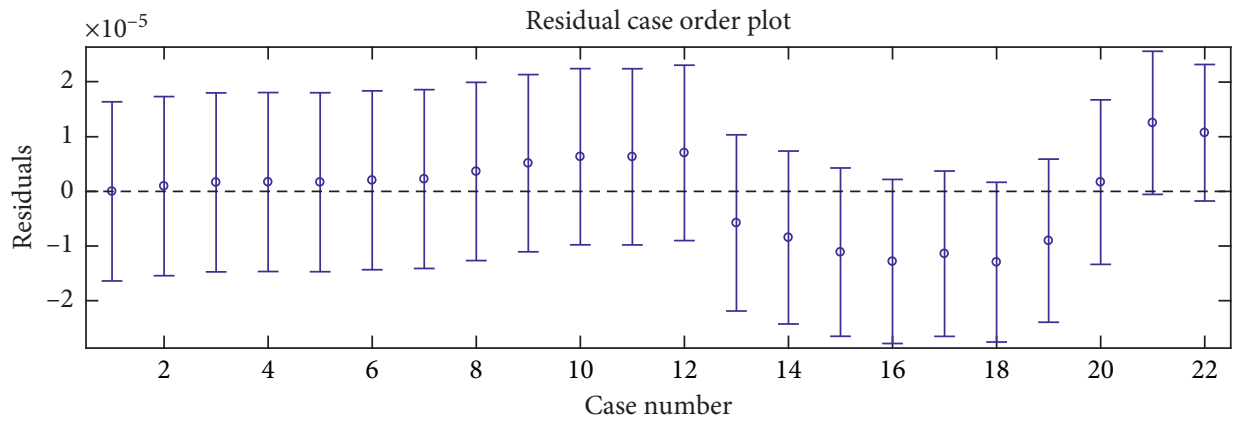

FIGURE 4: 95\% confidence intervals of residuals for infectious proportions.

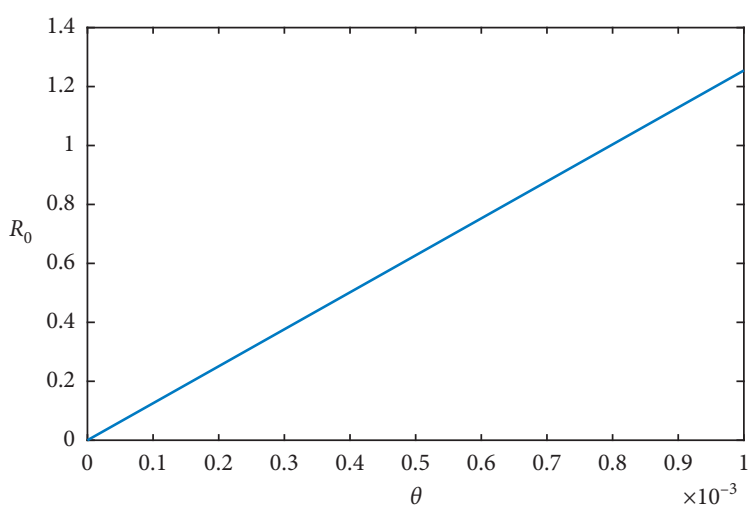

(a)

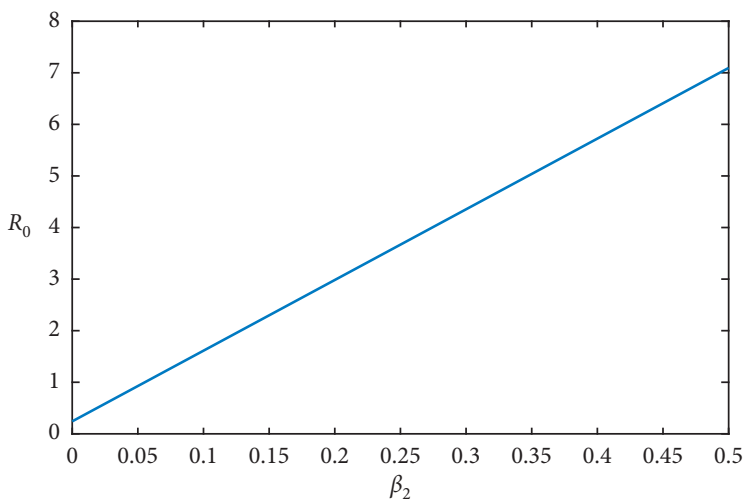

(c)

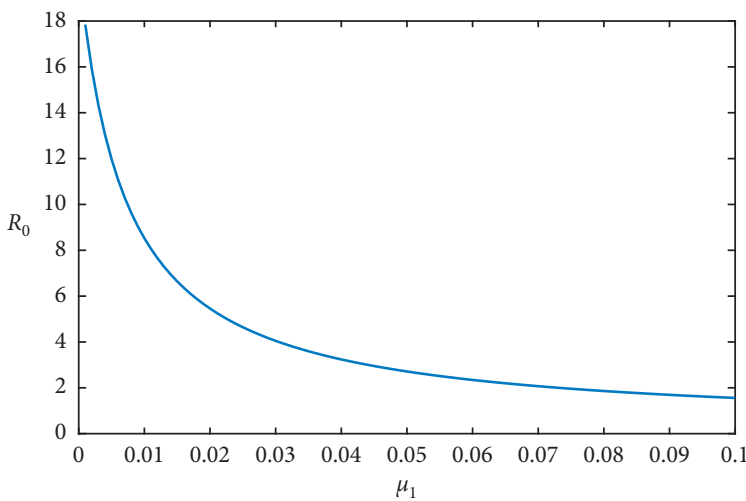

(e)

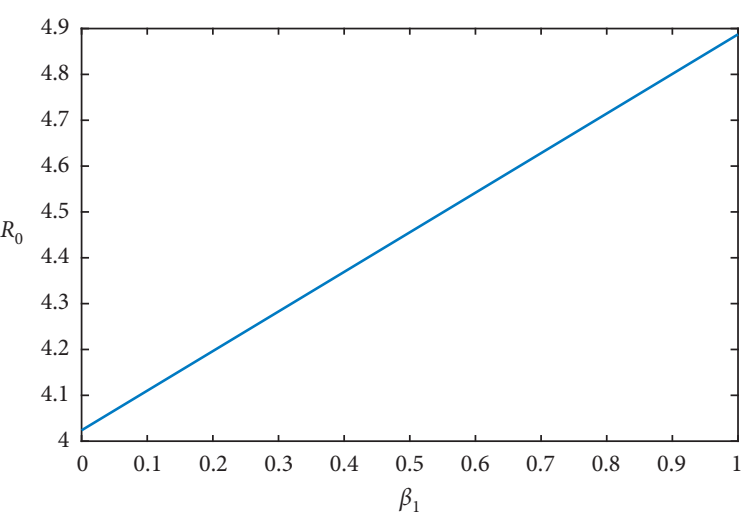

(b)

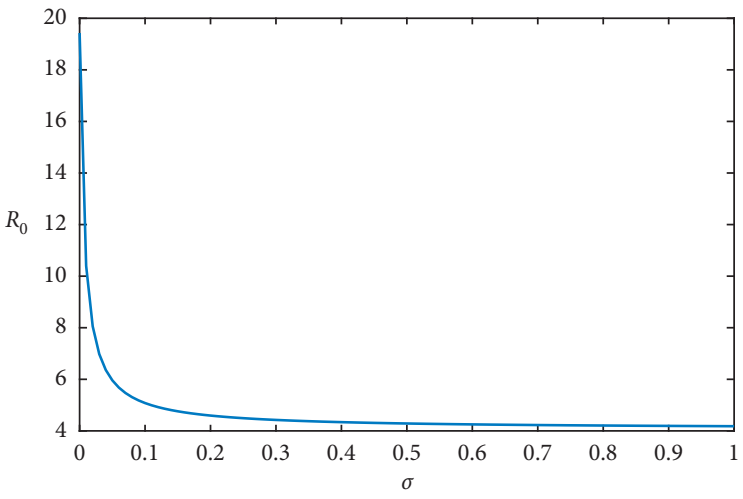

(d)

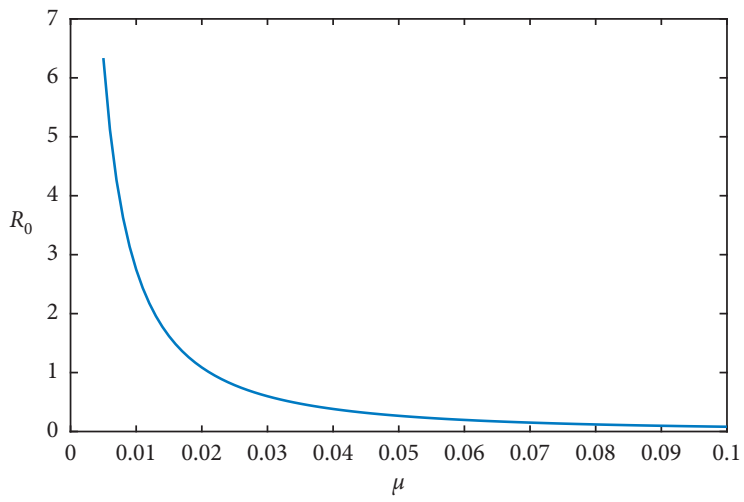

(f)

FiguRE 5: Effects of six parameters for $R_{0}$ under all other parameters given. (a) $\beta_{1}=0.2800, \beta_{2}=0.2936, \sigma=0.5554, \mu=$ 0.007 , and $\mu_{1}=0.0280 ; \quad$ (b) $\theta=0.0034, \beta_{2}=0.2936, \sigma=0.5554, \mu=0.007$, and $\mu_{1}=0.0280 ; \quad$ (c) $\theta=0.0034, \beta_{1}=0.2800, \sigma=0.5554, \mu=$ 0.007 , and $\mu_{1}=0.0280 ; \quad$ (d) $\theta=0.0034, \beta_{1}=0.2800, \beta_{2}=0.2936, \mu=0.007$, and $\mu_{1}=0.0280 ; \quad$ (e) $\theta=0.0034, \beta_{1}=0.2800, \beta_{2}=0.2936, \sigma=$ 0.5554 , and $\mu=0.007$; (f) $\theta=0.0034, \beta_{1}=0.2800, \beta_{2}=0.2936, \sigma=0.5554$, and $\mu_{1}=0.0280$. 


$$
\begin{aligned}
\zeta_{\theta} & =1 \\
\zeta_{\beta_{1}} & =\frac{\beta_{1}\left(\mu+\mu_{1}\right)}{\beta_{2} \sigma+\beta_{1}\left(\mu+\mu_{1}\right)}, \\
\zeta_{\beta_{2}} & =\frac{\beta_{2} \sigma}{\beta_{2} \sigma+\beta_{1}\left(\mu+\mu_{1}\right)}, \\
\zeta_{\sigma} & =\frac{\beta_{2} \sigma(\mu+\sigma)-\beta_{1} \sigma\left(\mu+\mu_{1}\right)-\beta_{2} \sigma^{2}}{\beta_{1}\left(\mu+\mu_{1}\right)(\mu+\sigma)+\beta_{2} \sigma(\mu+\sigma)}, \\
\zeta_{\mu_{1}} & =-\frac{\beta_{2} \mu_{1} \sigma}{\beta_{1}\left(\mu+\mu_{1}\right)^{2}+\beta_{2} \sigma\left(\mu+\mu_{1}\right)}, \\
\zeta_{\mu} & =-\frac{\left(\beta_{1}\left(\mu+\mu_{1}\right)+\beta_{2} \sigma\right)\left(\mu+\mu_{1}\right)(2 \mu+\sigma)+\beta_{2} \mu \sigma(\mu+\sigma)}{\left(\mu+\mu_{1}\right)(\mu+\sigma)\left(\beta_{1}\left(\mu+\mu_{1}\right)+\beta_{2} \sigma\right)} .
\end{aligned}
$$

For instance, take $\beta_{1}=0.2800, \beta_{2}=0.2936, \sigma=$ $0.5554, \mu_{1}=0.00280$, and $\mu=0.007$, then $R_{0}=1254.588 \theta$, where the value 1254.588 is the effect of all other parameters. Thus, $\zeta_{\theta}=(1 / 1254.588)\left(\mathrm{d} R_{0} / \mathrm{d} \theta\right)=1$. According to Table 2 and the above formulas, the sensitivity indexes of $R_{0}$ with respect to those parameters are listed in Table 3 . The sensitivity analysis demonstrates that $\theta, \beta_{2}, \mu_{1}$, and $\mu$ are highly sensitive to changes in the value of $R_{0}$. Moreover, decreasing $\theta$ and $\beta_{i}(i=1,2)$ means decreasing $R_{0}$, different from $\sigma, \mu_{1}$, and $\mu$.

3.3. Dynamical and Diffusion Analyses. In this section, we mainly investigate dynamical properties of $\mathbf{x}(t)$ and diffusion approximation of density process $\left\{\mathbf{X}_{N}(t): t \geq 0\right\}$ in (21) when $R_{0}>1$.

According to the results of Section 2.2, $(S(t), E(t)$, $I(t))=\left(N x_{1}(t), N x_{2}(t), N x_{3}(t)\right)$, where $x_{i}(t)(i=1,2,3)$ is defined in (6). Since the endemic equilibrium $\mathbf{x}^{*}=(0.1139,0.0046,0.0734)$, we have $N \mathbf{x}^{*}=(6739463$, $272182,4343078)$. By Theorem 2, $(S(t), E(t), I(t)) \longrightarrow$
TABLE 3: Sensitivity indexes of $R_{0}$ about different parameters.

\begin{tabular}{lc}
\hline Parameter & Sensitivity index \\
\hline$\theta$ & 1.0000 \\
$\beta_{1}$ & 0.0567 \\
$\beta_{2}$ & 0.9433 \\
$\sigma$ & -0.0442 \\
$\mu_{1}$ & -0.7546 \\
$\mu$ & -1.2011 \\
\hline
\end{tabular}

$(6739463,272182,4343078)$ as $t \longrightarrow+\infty$. Figure 6 provides the dynamical behavior of $(S(t), E(t), I(t))$.

On the other hand, from (21), the process $\left(S_{N}(t), E_{N}(t), I_{N}(t)\right) \mid I_{N}(t)>0 \sim N\left(N \mathbf{x}^{*}, N \Sigma\right), \quad$ where $\partial F\left(\mathbf{x}^{*}\right) \Sigma+\Sigma \partial F\left(\mathbf{x}^{*}\right)^{T}=-G\left(\mathbf{x}^{*}\right)$. After calculation, we have

$$
\begin{aligned}
\partial F\left(\mathbf{x}^{*}\right) & =\left(\begin{array}{ccc}
-\widehat{\beta}_{1} x_{2}^{*}-\widehat{\beta}_{2} x_{3}^{*}-\widehat{\mu} & -\widehat{\beta}_{1} x_{1}^{*} & -\widehat{\beta}_{2} x_{1}^{*} \\
\widehat{\beta}_{1} x_{2}^{*}+\widehat{\beta}_{2} x_{3}^{*} & \widehat{\beta}_{1} x_{1}^{*}-(\widehat{\mu}+\widehat{\sigma}) & \widehat{\beta}_{2} x_{1}^{*} \\
0 & \widehat{\sigma} & -\left(\widehat{\mu}+\widehat{\mu}_{1}\right)
\end{array}\right) \\
& =\left(\begin{array}{ccc}
-0.0298 & -0.0319 & -0.0334 \\
0.0228 & -0.5305 & 0.0334 \\
0 & 0.5554 & -0.0350
\end{array}\right) .
\end{aligned}
$$

Similarly, by (17), it is easy to get

$$
G\left(\mathbf{x}^{*}\right)=\left(\begin{array}{ccc}
0.0068 & -0.0026 & 0 \\
-0.0026 & 0.0052 & -0.0026 \\
0 & -0.0026 & 0.0051
\end{array}\right)
$$

Then,

$$
\Sigma=\left(\begin{array}{ccc}
0.2418 & -0.0022 & -0.1120 \\
-0.0022 & 0.0052 & 0.0064 \\
-0.1120 & 0.0064 & 0.1753
\end{array}\right) \text {. }
$$

From (21) and $R_{0}>1$,

$$
\left(S_{N}(t), E_{N}(t), I_{N}(t)\right) \sim N\left((6739463,272182,4343078),\left(\begin{array}{ccc}
14307306 & -130174 & -6627042 \\
-130174 & 307684 & 378688 \\
-6627042 & 378688 & 10372501
\end{array}\right)\right)
$$

Figure 7(a) shows the approximation of the quasi-stationary distribution with a trajectory simulated from (29). In Figures $7(\mathrm{~b})-7(\mathrm{~d})$, they provide the approximation marginal trajectories of the susceptible, exposed, and infectious individuals against time, respectively.

3.4. Control Strategies. Note that dynamical and diffusion properties of the deterministic model and density process are based on larger $N$ and $t$ tending to infinity. In practice, however, some good control strategies have been applied to prevent the spread of 2019-nCoV epidemic such as input control, screening, and isolation for treatment in Hubei province. Let $c_{i}(i=1,2,3)$ be control variables about input, screening, and isolation for treatment in order to reduce input rate $\theta$ and contact rates $\beta_{i}(i=1,2)$. When these control variables are introduced into model (6), we have 


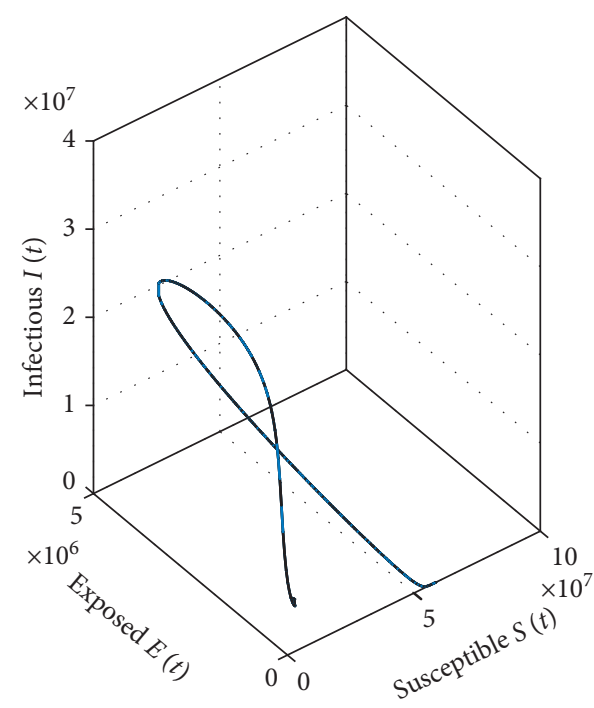

(a)

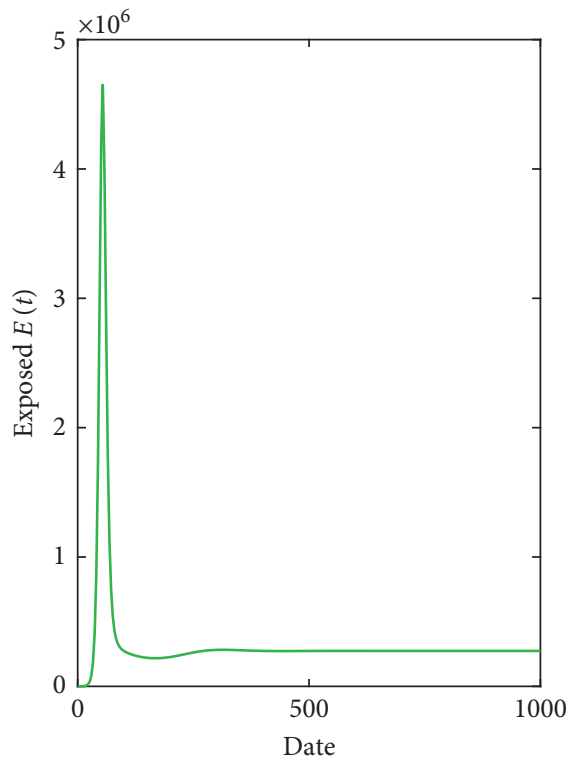

(c)

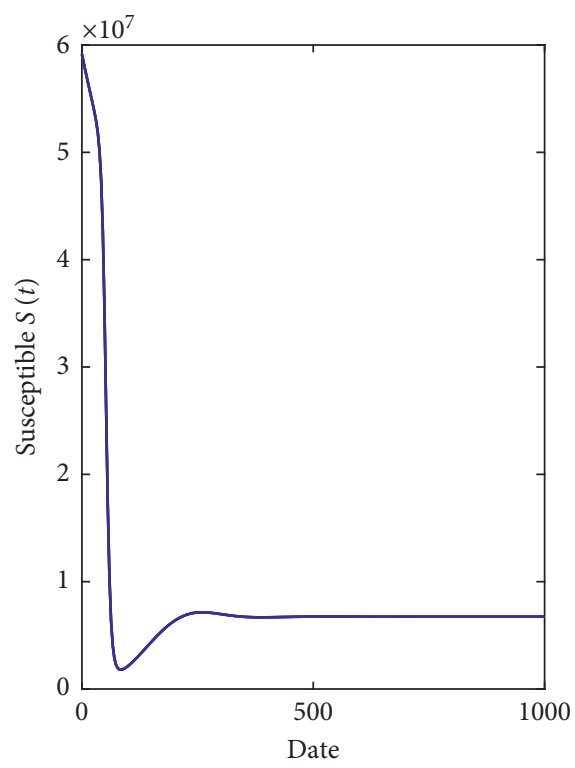

(b)

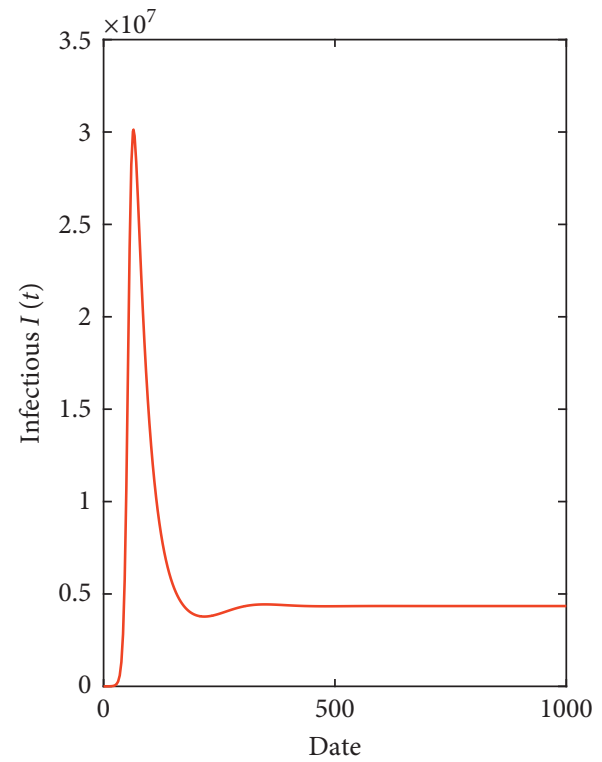

(d)

Figure 6: Dynamical behaviors of the susceptible, exposed, and infectious individuals.

$$
\left\{\begin{array}{l}
\frac{\mathrm{d} x_{1}(t)}{\mathrm{d} t}=\left(\theta-c_{1}\right)-\left(\beta_{1}-c_{2}\right) x_{1}(t) x_{2}(t)-\left(\beta_{2}-c_{3}\right) x_{1}(t) x_{3}(t)-\mu x_{1}(t), \\
\frac{\mathrm{d} x_{2}(t)}{\mathrm{d} t}=\left(\beta_{1}-c_{2}\right) x_{1}(t) x_{2}(t)+\left(\beta_{2}-c_{3}\right) x_{1}(t) x_{3}(t)-(\mu+\sigma) x_{2}(t), \\
\frac{\mathrm{d} x_{3}(t)}{\mathrm{d} t}=\sigma x_{2}(t)-\left(\mu+\mu_{1}\right) x_{3}(t) .
\end{array}\right.
$$

Under different control settings, we calculate the endemic equilibrium $\mathbf{x}^{*}$ and $R_{0}$ according to four cases:
(I) Input control variable $c_{1}>0$ and other variables $c_{2}=c_{3}=0$. 


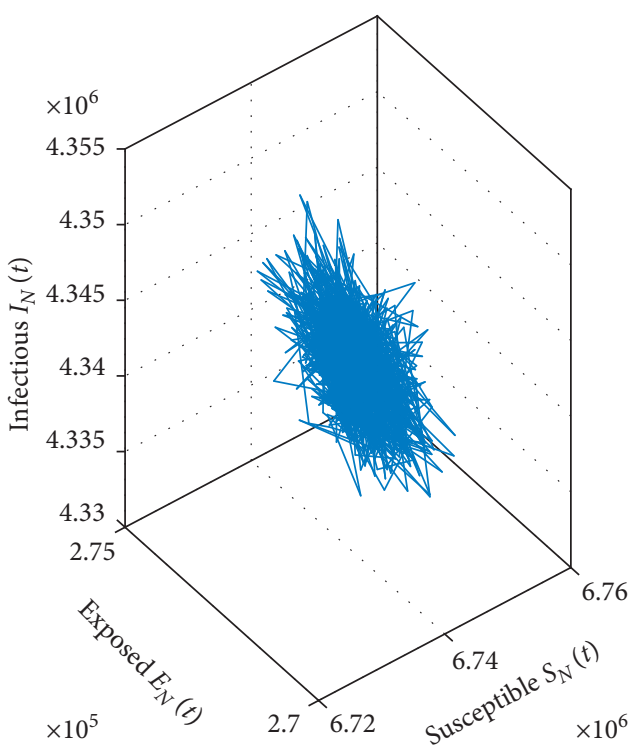

(a)

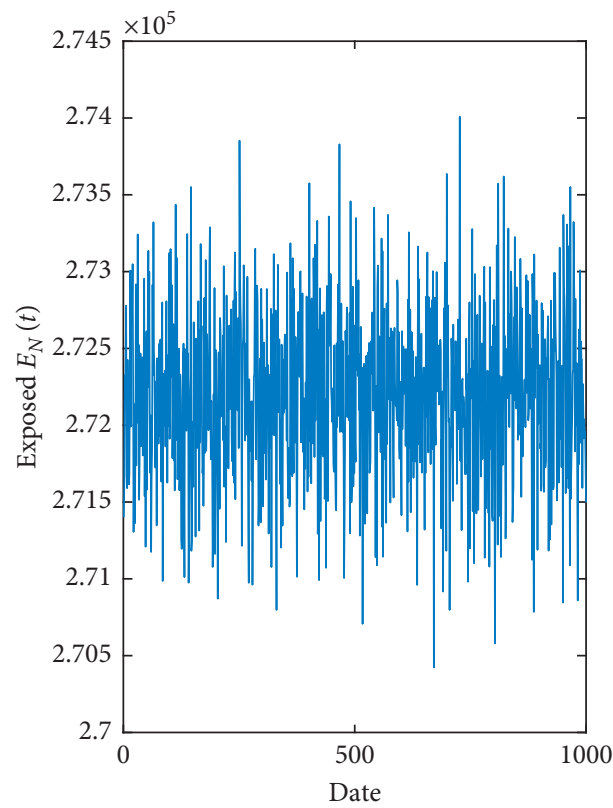

(c)

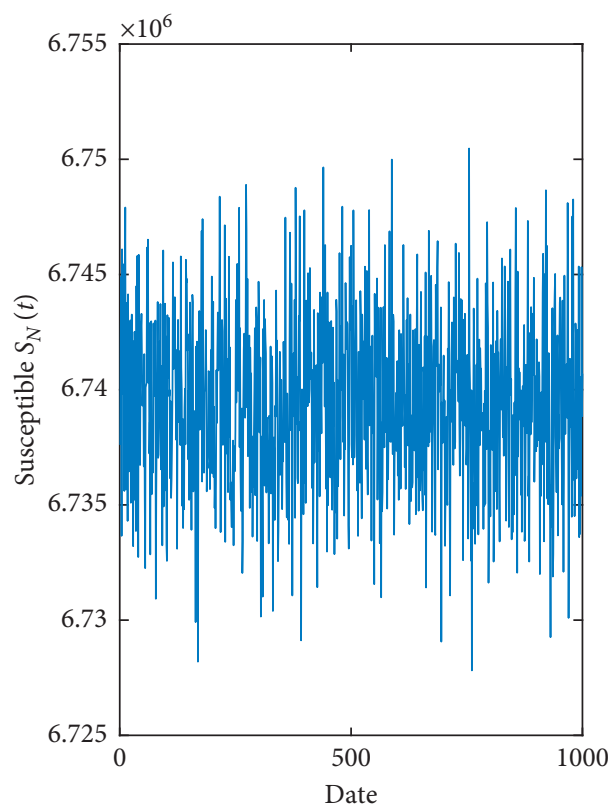

(b)

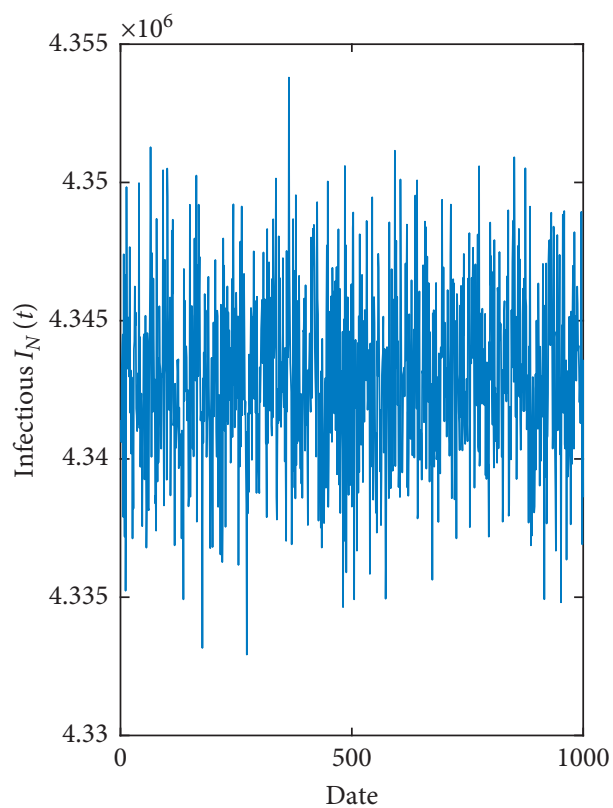

(d)

Figure 7: (a) Approximation of the quasi-stationary distribution; (b) marginal density of the susceptible; (c) marginal density of the exposed; (d) marginal density of infectious individuals.

(II) Screening control variable $c_{2}>0$ and other variables $c_{1}=c_{3}=0$.

(III) Isolation and treatment variable $c_{3}>0$ and other variables $c_{1}=c_{2}=0$.

(IV) All control variables are nonzero.

Comparing with $\mathbf{x}^{*}$ and $R_{0}$ of these cases $\mathrm{I}_{0}^{*}$-IV in Table 4, the control strategies III (d) and IV (d) are better than other strategies. On the other hand, we observe that the strategy IV (d) not only has a relatively satisfactory equilibrium $\mathbf{x}^{*}$ but also produces the smallest $R_{0}$. Thus, the use of strategy IV (d) is suggested for general application. Based on Table 4, dynamical properties of deterministic model (30) and diffusion properties of its density process can be obtained, similar to model (6) and (21). The detailed procedure will not be described here.

\section{Discussions}

In this paper, we propose an SEI epidemic process and a deterministic proportion model to investigate the outbreak of 2019-nCoV epidemic from 15 January 2020 to 5 February 
TABLE 4: The endemic equilibrium $\mathbf{x}^{*}$ and $R_{0}$ under different control variables $c_{i}(i=1,2,3)$.

\begin{tabular}{|c|c|c|c|c|c|c|}
\hline Case & Control strategy & Control variable $c_{1}$ & Control variable $c_{2}$ & Control variable $c_{3}$ & $\mathbf{x}^{*}$ & $R_{0}$ \\
\hline \multirow[t]{2}{*}{$I_{0}^{*}$} & - & 0 & 0 & 0 & $(0.1139,0.0046,0.0734)$ & 4.2656 \\
\hline & (a) & 0.0002 & 0 & 0 & $(0.1139,0.0043,0.0678)$ & 4.0146 \\
\hline \multirow{3}{*}{ I } & (b) & 0.0004 & 0 & 0 & $(0.1139,0.0039,0.0622)$ & 3.7637 \\
\hline & (c) & 0.0014 & 0 & 0 & $(0.1139,0.0021,0.0339)$ & 2.5092 \\
\hline & (d) & 0.0026 & 0 & 0 & $(0.1139,0.0000,0.0001)$ & 1.0037 \\
\hline \multirow{4}{*}{ II } & (a) & 0 & 0.0300 & 0 & $(0.1146,0.0046,0.0733)$ & 4.2396 \\
\hline & (b) & 0 & 0.1800 & 0 & $(0.1182,0.0046,0.0726)$ & 4.1101 \\
\hline & (c) & 0 & 0.2300 & 0 & $(0.1194,0.0046,0.0723)$ & 4.0669 \\
\hline & (d) & 0 & 0.2800 & 0 & $(0.1207,0.0045,0.0721)$ & 4.0237 \\
\hline \multirow{4}{*}{ III } & (a) & 0 & 0 & 0.0936 & $(0.1628,0.0040,0.0638)$ & 2.9828 \\
\hline & (b) & 0 & 0 & 0.1636 & $(0.2400,0.0031,0.0485)$ & 2.0234 \\
\hline & (c) & 0 & 0 & 0.1936 & $(0.3013,0.0023,0.0364)$ & 1.6123 \\
\hline & (d) & 0 & 0 & 0.2336 & $(0.4565,0.0004,0.0058)$ & 1.0641 \\
\hline \multirow{4}{*}{ IV } & (a) & 0.0001 & 0.0100 & 0.0436 & $(0.1327,0.0042,0.0669)$ & 3.5518 \\
\hline & (b) & 0.0002 & 0.0200 & 0.0936 & $(0.1638,0.0037,0.0579)$ & 2.7911 \\
\hline & (c) & 0.0003 & 0.0800 & 0.1936 & $(0.3147,0.0016,0.0253)$ & 1.4070 \\
\hline & (d) & 0.0004 & 0.0940 & 0.2226 & $(0.4284,0.0000,0.0000)$ & 1.0003 \\
\hline
\end{tabular}

Note: the case $I_{0}^{*}$ corresponds to the results of Table 2 .

2020 in Hubei province, China. In order to understand the property of the SEI process in endemic phase, the quasistationary distribution is of additional importance. An approximation approach is used to analyze quasi-stationary distribution based on the deterministic model.

Firstly, by scale transformation, the SEI process is equivalent to a density process with transition rate. Theorem 1 provides the relationship between the density process and the proportion model. Then, the basic reproduction number $R_{0}$ and endemic equilibrium are given by the proportion model. Theorem 2 reveals that the density process converges uniformly in probability to the endemic equilibrium. However, it cannot reflect the fluctuations of the density process around the endemic equilibrium. To get a more accurate approximation, we make use of the Gaussian diffusion process to approximate quasi-stationary distribution (Theorem 3). Further, it behaves like a three-dimensional $\mathrm{O}-\mathrm{U}$ process, fluctuating around the endemic equilibrium of the deterministic model under $R_{0}>1$.

The 2019-nCoV data of Hubei province are conducted to evaluate the performance of the proposed models. Leastsquares method is used to estimate unknown parameters, and residual analysis provides measures of model quality. Since $R_{0}$ is an important value to predict the spread of 2019nCoV epidemic, sensitivity analysis reveals that $R_{0}$ will decrease with smaller input rate and contact rates. Further, the sensitivity index of each parameter is given. Based on the above-estimated values, dynamical properties of the proportion model and diffusion approximation of density process are obtained for a long time, respectively. Since $R_{0}=4.2656>1$, from 15 January 2020 to 5 February 2020, 2019-nCoV infection will continue to spread and be endemic in long term if there is no effective control strategy in Hubei province (Figures 6 and 7). In order to prevent the 2019nCoV epidemic, some good control strategies are carried out. Three control variables are introduced into the proportion model. Table 4 reveals that the control strategy IV (d) is an optimal design, compared with other strategies. The strategy has not only a relative satisfactory equilibrium but also the smallest $R_{0}$. Thus, input control, screening, and isolation for treatment are of vital importance to prevent the spread of 2019-nCoV epidemic in Hubei province. In the practice, it has proved that the strategy is really feasible and effective to prevent the spread of 2019-nCoV epidemic.

The work in this article bears some limitations and concerns. For instance, the time to disease extinction for $2019-\mathrm{nCoV}$ is worth pursuing. Moreover, when $R_{0}$ is less than 1 or equal to 1 , the distribution can be approximately geometric or other distributions. For the problems, we will leave these for future consideration.

\section{Data Availability}

The data used to support the results of this study are available from the National Health Commission of the People's Republic of China.

\section{Conflicts of Interest}

The authors declare that there are no conflicts of interest regarding the publication of this article.

\section{Acknowledgments}

This research was supported by the National Natural Science Foundation of China (Grant no. 11661076) and the Science and Technology Department of Xinjiang Uygur Autonomous Region (Grant no. 2018Q011).

\section{References}

[1] Wuhan Municipal Health Commission, Notification of Viral Pneumonia of Unknown Cause, Wuhan Municipal Health Commission, Wuhan, China, 2020, http://wjw.wuhan.gov.cn/ front/web/showDetail/2020010309017. 
[2] Wuhan Munipical Health Commission, Information on the Current Situation of Pneumonia in Our City, Wuhan Munipical Health Commission, Wuhan, China, 2020, http:// wjw.wuhan.gov.cn/front/web/showDetail/2019123108989.

[3] Health Emergency Office, The Latest Situation of Pneumonia Caused by Novel Coronavirus Infection was Reported, 2020, http://www.nhc.gov.cn/xcs/yqfkdt/202002/ 4 f28ab5ca87d42d284833df3ccc8d45a.shtml.

[4] World Health Organization, Novel Coronavirus-China, World Health Organization, Geneva, Switzerland, 2020, https:// www.who.int/csr/don/12-january-2020-novel-coronaviruschina/en/.

[5] National Health Commission of the Peoples Republic China, Announcement No. 12019 Coronavirus Infected Pneumonia to Be Included in the Management of Statutory Infectious Diseases in 2020, National Health Commission of the Peoples Republic China, Beijing, China, 2020, http://www.nhc.gov.cn/jkj/s7916/ 202001/44a3b8245e8049d2837a4f27529cd386.shtml.

[6] New York Post, World Health Organization Gives New Coronavirus Name: COVID-19, New York Post, New York, NY, USA, 2020, https://nypost.com/2020/02/11/worldhealth-organization-gives-new-coronavirus-name-covid-19/.

[7] J. A. Backer, D. Klinkenberg, and J. Wallinga, "Incubation period of 2019-nCoV coronavirus (2019-nCoV) infections among travellers from Wuhan, China," Euro Surveill, vol. 25, no. 5, 2020 .

[8] T. Chen, J. Rui, Q. Wang et al., "A mathematical model for simulating the transmission of Wuhan novel coronavirus," BioRxiv, 2020.

[9] Q. Lin, S. Zhao, D. Gao et al., "A conceptual model for the coronavirus disease 2019 (COVID-19) outbreak in Wuhan, China with individual reaction and governmental action," International Journal of Infectious Diseases, vol. 93, pp. 211-216, 2020.

[10] J. T. Wu, K. Leung, and G. M. Leung, "Nowcasting and forecasting the potential domestic and international spread of the 2019-nCoV outbreak originating in Wuhan, China: a modelling study," The Lancet, vol. 395, no. 10225, pp. 689697, 2020.

[11] Q. Cui, Z. Hu, Y. Li, J. Han, Z. Teng, and J. Qian, "Dynamic variations of the COVID-19 disease at different quarantine strategies in Wuhan and mainland China," Journal of Infection and Public Health, vol. 13, no. 6, pp. 849-855. In press, 2020.

[12] Z. Hu, Q. Cui, J. Han, X. Wang, W. E. I. Sha, and Z. Teng, "Evaluation and prediction of the COVID-19 variations at different input population and quarantine strategies, a case study in Guangdong province, China," International Journal of Infectious Diseases, vol. 95, pp. 231-240, 2020.

[13] W. K. Zhou, A. L. Wang, F Xia, Y. N Xiao, and S. Y Tang, "Effects of media reporting on mitigating spread of COVID19 in the early phase of the outbreak," Mathematical Biosciences and Engineering: MBE, vol. 17, no. 3, pp. 2693-2707, 2020.

[14] C. D. Russell, J. E. Millar, and J. K. Baillie, "Clinical evidence does not support corticosteroid treatment for 2019-nCoV lung injury," The Lancet, vol. 395, no. 10223, pp. 473-475, 2020.

[15] C. del Rio and P. N. Malani, "2019 novel coronavirus-important information for clinicians," JAMA, vol. 323, no. 11, p. 1039, 2020.

[16] X. He, L. Zhang, Q. Ran et al., "Integrative bioinformatics analysis provides insight into the molecular mechanisms of 2019-nCoV," MedRxiv, 2020.
[17] J. F. Chan, S. Yuan, K. KoK et al., "A familial cluster of pneumonia associated with the 2019 novel coronavirus indicating person-to-person transmission: a study of a family cluster," The Lancet, vol. 395, no. 10223, pp. 514-523, 2020.

[18] B. Tang, X. Wang, Q. Li et al., "Estimation of the transmission risk of the 2019-nCoV and its implication for public health interventions," Journal of Clinical Medicine, vol. 9, no. 2, p. 462, 2020.

[19] S. Zhao, S. S. Musa, Q. Lin et al., "Estimating the unreported number of novel coronavirus $(2019-\mathrm{nCoV})$ cases in China in the first half of January 2020: a data-driven modelling analysis of the early outbreak," Journal of Clinical Medicine, vol. 9, no. 2, p. 388, 2020.

[20] S. Zhao, Q. Lin, J. Ran et al., "Preliminary estimation of the basic reproduction number of novel coronavirus (2019$\mathrm{nCoV}$ ) in China, from 2019 to 2020: a data-driven analysis in the early phase of the outbreak," International Journal of Infectious Diseases, vol. 92, pp. 214-217, 2020.

[21] I. Nåsell, "Stochastic models of some endemic infections," Mathematical Biosciences, vol. 179, no. 1, pp. 1-19, 2002.

[22] D. Zhao and S. Yuan, "Persistence and stability of the diseasefree equilibrium in a stochastic epidemic model with imperfect vaccine," Advances in Difference Equations, vol. 2016, no. 1, 2016.

[23] H. W. Hethcote, "The mathematics of infectious diseases," SIAM Review, vol. 42, no. 4, pp. 599-653, 2000.

[24] R. Fierro, "A class of stochastic epidemic models and its deterministic counterpart," Journal of the Korean Statistical Society, vol. 39, no. 4, pp. 397-407, 2010.

[25] J. Chen, "Pathogenicity and transmissibility of 2019-nCoV-a quick overview and comparison with other emerging viruses," Microbes and Infection, vol. 22, no. 2, pp. 69-71, 2020.

[26] E. A. Van Doorn and P. K. Pollett, "Quasi-stationary distributions," in Memorandum 1945, Department of Applied Mathematics, University of Twente, Enschede, Netherlands, 2011, http://www.math.utwente.nl/publictions.

[27] I. Nåsell, "On the quasi-stationary distribution of the stochastic logistic epidemic," Mathematical Biosciences, vol. 156, no. 1-2, pp. 21-40, 1999.

[28] J. V. Ross, T. Taimre, and P. K. Pollett, "On parameter estimation in population models," Theoretical Population Biology, vol. 70, no. 4, pp. 498-510, 2006.

[29] T. G. Kurtz, "Approximation of population processes," in CBMS-NSF Regional Conference Series in Applied Mathematics, Vol. 36, Society for Industrial and Applied Mathematics, Philadelphia, PA, USA, 1981.

[30] A. D. Barbour, "On a functional central limit theorem for Markov population processes," Advances in Applied Probability, vol. 6, no. 1, pp. 21-39, 1974.

[31] News Press and Situation Reports of the Pneumonia Caused by Novel Coronavirus, 2020, http://wjw.wuhan.gov.cn/front/ web/list2nd/no/710.

[32] An Outbreak Situation Update on the Pneumonia Caused by the Novel Coronavirus (2019-nCoV) Infection, 2020, http:// www.nhc.gov.cn/xcs/yqtb/list_gzbd.shtml.

[33] National Bureau of Statistics of China, 2020, http://data.stats. gov.cn/easyquery.htm?cn=E0103. 